LINEAR AND NON-LINEAR THEORY OF

GENERALIZED FUNCTIONS AND ITS APPLICATIONS

BANACH CENTER PUBLICATIONS, VOLUME 88

INSTITUTE OF MATHEMATICS

POLISH ACADEMY OF SCIENCES

WARSZAWA 2010

\title{
FOURIER-WIGNER TRANSFORMS AND LIOUVILLE'S THEOREMS FOR THE SUB-LAPLACIAN ON THE HEISENBERG GROUP
}

\author{
APARAJITA DASGUPTA and M. W. WONG \\ Department of Mathematics and Statistics, York University \\ 4700 Keele Street, Toronto, Ontario M3J 1P3, Canada \\ E-Mail:adgupta@mathstat.yorku.ca,mwwong@mathstat.yorku.ca
}

\begin{abstract}
The sub-Laplacian on the Heisenberg group is first decomposed into twisted Laplacians parametrized by Planck's constant. Using Fourier-Wigner transforms so parametrized, we prove that the twisted Laplacians are globally hypoelliptic in the setting of tempered distributions. This result on global hypoellipticity is then used to obtain Liouville's theorems for harmonic functions for the sub-Laplacian on the Heisenberg group.
\end{abstract}

1. The sub-Laplacian on the Heisenberg group. The aim of this paper is to study the growth properties of harmonic functions for the sub-Laplacian $\mathcal{L}$ on the Heisenberg group $\mathbb{H}$. The main results are seen in the perspective of Liouville's theorems for the sub-Laplacian $\mathcal{L}$ on the Heisenberg group $\mathbb{H}$. We first decompose the sub-Laplacian $\mathcal{L}$ into a family of twisted Laplacians $L_{\tau}$ on $\mathbb{C}, \tau \in \mathbb{R} \backslash\{0\}$. Then using $\tau$-Fourier-Wigner transforms of $\tau$-Hermite functions that we define in Section 3, we give formulas for the heat kernel and Green function of the twisted Laplacian $L_{\tau}$ for every $\tau$ in $\mathbb{R} \backslash\{0\}$. These formulas are analogues of the ones for $L_{1}$, which can be found in [15. The Green function of $L_{\tau}$ is then used to prove that $L_{\tau}$ is globally hypoelliptic in the setting of tempered distributions as in [15]. Using the global hypoellipticity of $L_{\tau}$ for every $\tau$ in $\mathbb{R} \backslash\{0\}$, the main results on the growth properties of harmonic functions for the sub-Laplacian $\mathcal{L}$ can be established easily.

Basic information on the classical Fourier-Wigner transforms can be found in 12 among others.

2000 Mathematics Subject Classification: Primary 47F05, 47G30; Secondary 35H20.

Key words and phrases: Heisenberg group, sub-Laplacian, twisted Laplacians, Hermite functions, Fourier-Wigner transforms, heat kernels, Green functions, global hypoellipticity, Liouville's theorems.

The paper is in final form and no version of it will be published elsewhere. 
In Section 2, we show how to transform the sub-Laplacian $\mathcal{L}$ to a family of twisted Laplacians $L_{\tau}$ on $\mathbb{C}$ parametrized by $\tau$ in $\mathbb{R} \backslash\{0\}$. This has the advantage of reducing the number of independent variables of the sub-Laplacian from three to two and can be seen as a method of descent and the parameter can be looked at as Planck's constant. The $\tau$-Fourier-Wigner transforms of $\tau$-Hermite functions are developed in Section 3, which can then be used in Section 4 to construct the heat kernel of $L_{\tau}$. The Green function of $L_{\tau}$ is constructed in Section 5, which is used to show that $L_{\tau}$ is globally hypoelliptic in the setting of tempered distributions. Liouville's theorems for the sub-Laplacian $\mathcal{L}$ on the Heisenberg group $\mathbb{H}$ are given in Section 6 .

In an attempt to make the paper as self-contained and accessible as possible, we first recall the basic formulas and notions pertaining to the sub-Laplacian on the Heisenberg group. If we identify $\mathbb{R}^{2}$ with the complex plane $\mathbb{C}$ via the obvious identification

$$
\mathbb{R}^{2} \ni(x, y) \leftrightarrow z=x+i y \in \mathbb{C},
$$

and we let

$$
\mathbb{H}=\mathbb{C} \times \mathbb{R},
$$

then $\mathbb{H}$ becomes a non-commutative group when equipped with the multiplication $\cdot$ given by

$$
(z, t) \cdot(w, s)=\left(z+w, t+s+\frac{1}{4}[z, w]\right), \quad(z, t),(w, s) \in \mathbb{H},
$$

where $[z, w]$ is the symplectic form of $z$ and $w$ defined by

$$
[z, w]=2 \operatorname{Im}(z \bar{w}) .
$$

Let $\mathfrak{h}$ be the Lie algebra of left-invariant vector fields on $\mathbb{H}$. Then a basis for $\mathfrak{h}$ is given by $X, Y$ and $T$, where

$$
\begin{aligned}
& X=\frac{\partial}{\partial x}+\frac{1}{2} y \frac{\partial}{\partial t}, \\
& Y=\frac{\partial}{\partial y}-\frac{1}{2} x \frac{\partial}{\partial t},
\end{aligned}
$$

and

$$
T=\frac{\partial}{\partial t}
$$

The sub-Laplacian $\mathcal{L}$ on $\mathbb{H}$ is defined by

$$
\mathcal{L}=-\left(X^{2}+Y^{2}\right) .
$$

A simple computation gives

$$
\mathcal{L}=-\Delta-\frac{1}{4}\left(x^{2}+y^{2}\right) \frac{\partial^{2}}{\partial t^{2}}+\left(x \frac{\partial}{\partial y}-y \frac{\partial}{\partial x}\right) \frac{\partial}{\partial t},
$$

where

$$
\Delta=\frac{\partial^{2}}{\partial x^{2}}+\frac{\partial^{2}}{\partial y^{2}}
$$

Details on the Heisenberg group $\mathbb{H}$ and the sub-Laplacian $\mathcal{L}$ can be found in [1, 10, 11]. 
Since the symbol $\sigma(\mathcal{L})$ of $\mathcal{L}$ is given by

$$
\sigma(\mathcal{L})(x, y, t ; \xi, \eta, \tau)=\left(\xi+\frac{1}{2} y \tau\right)^{2}+\left(\eta-\frac{1}{2} x \tau\right)^{2}
$$

for all $(x, y, t)$ and $(\xi, \eta, \tau)$ in $\mathbb{R}^{3}$, it is easy to see that $\mathcal{L}$ is a nowhere elliptic partial differential operator on $\mathbb{R}^{3}$. Since

$$
[X, Y]=T,
$$

it follows from a well-known result of Hörmander in [7] that $\mathcal{L}$ is hypoelliptic. It is worth pointing out in this connection that the bracket generating hypothesis in Hörmander's theorem is exactly the same as the hypothesis in Chow's connectivity theorem in [4, which in the setting of the Heisenberg group $\mathbb{H}$ tells us that any two points in $\mathbb{H}$ can be connected by a horizontal smooth curve $c$, i.e., the vector field $\dot{c}$ lies in the span of the so-called horizontal vector fields $X$ and $Y$ on $\mathbb{H}$. Details can be found in $[3]$.

A function $u$ on $\mathbb{H}$ is said to be harmonic for the sub-Laplacian $\mathcal{L}$ if

$$
(\mathcal{L} u)(z, t)=0, \quad(z, t) \in \mathbb{H} .
$$

2. Twisted Laplacians. Let $\frac{\partial}{\partial z}$ and $\frac{\partial}{\partial \bar{z}}$ be partial differential operators on $\mathbb{R}^{2}$ given by

$$
\frac{\partial}{\partial z}=\frac{\partial}{\partial x}-i \frac{\partial}{\partial y}
$$

and

$$
\frac{\partial}{\partial \bar{z}}=\frac{\partial}{\partial x}+i \frac{\partial}{\partial y}
$$

Let $\tau \in \mathbb{R} \backslash\{0\}$. Then we define the partial differential operators $Z_{\tau}$ and $\bar{Z}_{\tau}$ by

$$
Z_{\tau}=\frac{\partial}{\partial z}+\frac{1}{2} \tau \bar{z}, \quad \bar{z}=x-i y
$$

and

$$
\bar{Z}_{\tau}=\frac{\partial}{\partial \bar{z}}-\frac{1}{2} \tau z, \quad z=x+i y .
$$

The partial differential operators $Z_{\tau}$ and $\bar{Z}_{\tau}$, and the identity operator $I$ form a basis for a Lie algebra in which the Lie bracket of two elements is their commutator. In fact, $-\bar{Z}_{\tau}$ is the formal adjoint of $Z_{\tau}$. Let $L_{\tau}$ be the partial differential operator on $\mathbb{R}^{2}$ defined by

$$
L_{\tau}=-\frac{1}{2}\left(Z_{\tau} \bar{Z}_{\tau}+\bar{Z}_{\tau} Z_{\tau}\right)
$$

Then $L_{\tau}$ is an elliptic partial differential operator on $\mathbb{R}^{2}$ given by

$$
L_{\tau}=-\Delta+\frac{1}{4}\left(x^{2}+y^{2}\right) \tau^{2}-i\left(x \frac{\partial}{\partial y}-y \frac{\partial}{\partial x}\right) \tau .
$$

Thus, $L_{\tau}$ is the ordinary Hermite operator $-\Delta+\frac{1}{4}\left(x^{2}+y^{2}\right) \tau^{2}$ perturbed by the partial differential operator $-i N \tau$, where

$$
N=x \frac{\partial}{\partial y}-y \frac{\partial}{\partial x}
$$

is the rotation operator. As such, we call $L_{\tau}$ the twisted Laplacian. If $\tau=1$, then we recover the twisted Laplacian studied in detail in [5, 13, 14, 15]. 
For every $\tau$ in $\mathbb{R} \backslash\{0\}$, the twisted Laplacian $L_{\tau}$ can be written as

$$
L_{\tau}=\left(D_{x}-\frac{1}{2} \tau y\right)^{2}+\left(D_{y}+\frac{1}{2} \tau x\right)^{2} .
$$

Its symbol $\sigma\left(L_{\tau}\right)$ is given by

$$
\sigma\left(L_{\tau}\right)(x, y ; \xi, \eta)=\left(\xi-\frac{1}{2} \tau y\right)^{2}+\left(\eta+\frac{1}{2} \tau x\right)^{2}
$$

for all $(x, y)$ and $(\xi, \eta)$ in $\mathbb{R}^{2}$. Thus, $L_{\tau}$ is elliptic, but not globally elliptic. It is not even globally elliptic in the sense defined in Section 25 of [9]. The globally hypoellipticity in the setting of tempered distributions of $L_{\tau}$ is made precise in Section 5 and is a consequence of the estimates on the Green function $G_{\tau}$ of $L_{\tau}$. Detailed discussions on global hypoellipticity of pseudo-differential operators can be found on page 70 of the book [2] and also in the paper [8].

Let $\tau \in \mathbb{R}$. Then we define for all every function $f$ in $L^{1}(\mathbb{H})$, the function $f^{\tau}$ on $\mathbb{C}$ by

$$
f^{\tau}(z)=(2 \pi)^{-1 / 2} \int_{-\infty}^{\infty} e^{i t \tau} f(z, t) d t, \quad z \in \mathbb{C},
$$

provided that the integral exists. $f^{\tau}(z)$ is in fact the inverse Fourier transform of $f(z, t)$ with respect to $t$ evaluated at $\tau$. It is to be noted that the Fourier transform $\hat{F}$ of a function $F$ in $L^{1}\left(\mathbb{R}^{n}\right)$ is defined by

$$
\hat{F}(\xi)=(2 \pi)^{-n / 2} \int_{\mathbb{R}^{n}} e^{-i x \cdot \xi} F(x) d x, \quad \xi \in \mathbb{R}^{n} .
$$

Let $u$ be a tempered distribution on $\mathbb{H}=\mathbb{C} \times \mathbb{R}$ such that $u$ is equal to a $C^{\infty}$ function on $\mathbb{C} \times \mathbb{R}$ and $\check{u}(z, \tau)$ is a tempered function of $\tau$ on $\mathbb{R}$ for each fixed $z$ in $\mathbb{C}$, where $\check{u}$ is the inverse Fourier transform of $u$ with respect to the "second" variable $t$. For almost all $\tau$ in $\mathbb{R}$, we define $u^{\tau}$ on $\mathbb{C}$ by

$$
u^{\tau}(z)=\check{u}(z, \tau), \quad z \in \mathbb{C} .
$$

Then the fundamental connection between the twisted Laplacians $L_{\tau}, \tau \in \mathbb{R} \backslash\{0\}$, and the sub-Laplacian $\mathcal{L}$ is given by

$$
(\mathcal{L} u)^{\tau}=L_{\tau} u^{\tau}
$$

for almost all $\tau$ in $\mathbb{R} \backslash\{0\}$. The equation (2.2) follows from the most basic properties of Fourier transforms and tempered distributions.

3. $\tau$-Fourier-Wigner transforms of $\tau$-Hermite functions. Let $f$ and $g$ be functions in the Schwartz space $\mathcal{S}(\mathbb{R})$ on $\mathbb{R}$. Then for $\tau$ in $\mathbb{R} \backslash\{0\}$, the $\tau$-Fourier-Wigner transform $V_{\tau}(f, g)$ of $f$ and $g$ is defined by

$$
V_{\tau}(f, g)(q, p)=(2 \pi)^{-1 / 2}|\tau|^{1 / 2} \int_{-\infty}^{\infty} e^{i \tau q y} f\left(y+\frac{p}{2}\right) \overline{g\left(y-\frac{p}{2}\right)} d y
$$

for all $q$ and $p$ in $\mathbb{R}$. In fact,

$$
V_{\tau}(f, g)(q, p)=|\tau|^{1 / 2} V(f, g)(\tau q, p), \quad q, p \in \mathbb{R},
$$

where $V(f, g)$ is the classical Fourier-Wigner transform of $f$ and $g$. 
For $\tau \in \mathbb{R} \backslash\{0\}$ and for $k=0,1,2, \ldots$, we define $e_{k, \tau}$ to be the function on $\mathbb{R}$ by

$$
e_{k, \tau}(x)=|\tau|^{1 / 4} e_{k}(\sqrt{|\tau|} x), \quad x \in \mathbb{R} .
$$

For $j, k=0,1,2, \ldots$, we define $e_{j, k, \tau}$ on $\mathbb{R}^{2}$ by

$$
e_{j, k, \tau}=V_{\tau}\left(e_{j, \tau}, e_{k, \tau}\right) \text {. }
$$

The connection between $\left\{e_{j, k, \tau}: j, k=0,1,2, \ldots\right\}$ and $\left\{e_{j, k}: j, k=0,1,2, \ldots\right\}$ studied in [15] is given by the following formula.

Theorem 3.1. For $\tau \in \mathbb{R} \backslash\{0\}$ and for $j, k=0,1,2, \ldots$,

$$
e_{j, k, \tau}(q, p)=|\tau|^{1 / 2} e_{j, k}\left(\frac{\tau}{\sqrt{|\tau|}} q, \sqrt{|\tau|} p\right), \quad q, p \in \mathbb{R} .
$$

Proof. For $\tau \in \mathbb{R} \backslash\{0\}$ and for $j, k=0,1,2, \ldots$,

$$
\begin{aligned}
e_{j, k, \tau}(q, p) & =V_{\tau}\left(e_{j, \tau}, e_{k, \tau}\right)(q, p) \\
& =(2 \pi)^{-1 / 2}|\tau|^{1 / 2} \int_{-\infty}^{\infty} e^{i \tau q y} e_{j, \tau}\left(y+\frac{p}{2}\right) \overline{e_{k, \tau}\left(y-\frac{p}{2}\right)} d y \\
& =(2 \pi)^{-1 / 2}|\tau| \int_{-\infty}^{\infty} e^{i \tau q y} e_{j}\left(\sqrt{|\tau|}\left(y+\frac{p}{2}\right)\right) \overline{e_{k}\left(\sqrt{|\tau|}\left(y-\frac{p}{2}\right)\right)} d y \\
& =(2 \pi)^{-1 / 2}|\tau|^{1 / 2} \int_{-\infty}^{\infty} e^{i \tau q y / \sqrt{|\tau|}} e_{j}\left(y+\sqrt{|\tau|} \frac{p}{2}\right) \overline{e_{k}\left(y-\sqrt{|\tau|} \frac{p}{2}\right)} d y \\
& =|\tau|^{1 / 2} e_{j, k}\left(\frac{\tau}{\sqrt{|\tau|}} q, \sqrt{|\tau| p}\right)
\end{aligned}
$$

for all $q$ and $p$ in $\mathbb{R}$.

THEOREM 3.2. $\left\{e_{j, k, \tau}: j, k=0,1,2, \ldots\right\}$ forms an orthonormal basis for $L^{2}\left(\mathbb{R}^{2}\right)$.

Theorem 3.2 follows from Theorem 3.1 and Theorem 21.2 in [12] to the effect that $\left\{e_{j, k}: j, k=0,1,2, \ldots\right\}$ is an orthonormal basis for $L^{2}\left(\mathbb{R}^{2}\right)$.

TheOREM 3.3. For $j, k=0,1,2, \ldots$,

$$
L_{\tau} e_{j, k, \tau}=(2 k+1)|\tau| e_{j, k, \tau} .
$$

Theorem 3.3 can be proved using Theorem 3.1 and Theorem 22.2 in [12] telling us that for $j, k=0,1,2, \ldots, e_{j, k}$ is an eigenfunction of $L_{1}$ corresponding to the eigenvalue $2 k+1$.

Now, for every real number $\lambda$, we introduce the twisted convolution $f *_{\lambda} g$ of two measurable functions $f$ and $g$ on $\mathbb{C}$ given by

$$
\left(f *_{\lambda} g\right)(z)=\int_{\mathbb{C}} f(z-w) g(w) e^{i \lambda[z, w]} d w, \quad z \in \mathbb{C},
$$

provided that the integral exists. Then we have the following formula, which is the main tool for the construction of the heat kernel of $L_{\tau}$.

TheOREM 3.4. For $\tau \in \mathbb{R} \backslash\{0\}$ and for nonnegative integers $\alpha, \beta$, $\mu$ and $\nu$,

$$
e_{\alpha, \beta, \tau} *_{-\tau / 4} e_{\mu, \nu, \tau}=(2 \pi)^{1 / 2}|\tau|^{-1 / 2} \delta_{\beta, \mu} e_{\alpha, \nu, \tau},
$$

where $\delta_{\beta, \mu}$ is the Kronecker delta. 
When $\tau=1$, the formula is the same as that in Theorem 4.1 in [15]. Theorem 3.4 can be proved using the formula for $\tau=1$ and Theorem 3.1.

4. The heat kernel of $L_{\tau}$. Using Theorem 3.3 and the spectral theorem, we get for all functions $f$ in $L^{2}\left(\mathbb{R}^{2}\right)$,

$$
e^{-u L_{\tau}} f=\sum_{k=0}^{\infty} \sum_{j=0}^{\infty} e^{-(2 k+1)|\tau| u}\left(f, e_{j, k, \tau}\right) e_{j, k, \tau}, \quad u>0,
$$

where $($,$) is the inner product in L^{2}\left(\mathbb{R}^{2}\right)$. So, for $u>0$,

$$
e^{-u L_{\tau}} f=\sum_{k=0}^{\infty} e^{-(2 k+1)|\tau| u} \sum_{j=0}^{\infty}\left(f, e_{j, k, \tau}\right) e_{j, k, \tau}
$$

and our first task is to compute $\sum_{j=0}^{\infty}\left(f, e_{j, k, \tau}\right) e_{j, k, \tau}$. To this end, we note that for $k=$ $0,1,2, \ldots$,

$$
\begin{aligned}
f *_{-\tau / 4} e_{k, k, \tau} & =\sum_{j=0}^{\infty} \sum_{l=0}^{\infty}\left(f, e_{j, l, \tau}\right) e_{j, l, \tau} *_{-\tau / 4} e_{k, k, \tau} \\
& =\sum_{j=0}^{\infty} \sum_{l=0}^{\infty}\left(f, e_{j, l, \tau}\right)(2 \pi)^{1 / 2}|\tau|^{-1 / 2} \delta_{l, k} e_{j, k, \tau} \\
& =(2 \pi)^{1 / 2}|\tau|^{-1 / 2} \sum_{j=0}^{\infty}\left(f, e_{j, k, \tau}\right) e_{j, k, \tau} .
\end{aligned}
$$

Hence, for $k=0,1,2, \ldots$,

$$
\sum_{j=0}^{\infty}\left(f, e_{j, k, \tau}\right) e_{j, k, \tau}=(2 \pi)^{-1 / 2}|\tau|^{1 / 2}\left(f *_{-\tau / 4} e_{k, k, \tau}\right) .
$$

Therefore

$$
e^{-u L_{\tau}} f=(2 \pi)^{-1 / 2}|\tau|^{1 / 2} \sum_{k=0}^{\infty} e^{-(2 k+1)|\tau| u} e_{k, k, \tau} *_{\tau / 4} f, \quad u>0 .
$$

Now, using Theorem 3.1 and Mehler's formula, we get for all $z=(q, p)$ in $\mathbb{C}$ and for $u>0$,

$$
\begin{aligned}
(2 \pi)^{-1 / 2}|\tau|^{1 / 2} & \sum_{k=0}^{\infty} e^{-(2 k+1)|\tau| u} e_{k, k, \tau}(q, p) \\
& =(2 \pi)^{-1 / 2}|\tau| e^{-|\tau| u} \sum_{k=0}^{\infty} e^{-2 k|\tau| u} e_{k, k}\left(\frac{\tau}{\sqrt{|\tau|}} q, \sqrt{|\tau| p}\right) \\
& =(2 \pi)^{-1}|\tau| e^{-|\tau| u} \frac{1}{1-e^{-2|\tau| u}} e^{-|\tau||z|^{2} \frac{1}{4} \frac{1+e^{-2|\tau| u}}{1-e^{-2|\tau| u}}} \\
& =\frac{1}{4 \pi} \frac{\tau}{\sinh (\tau u)} e^{-\frac{1}{4}|\tau||z|^{2} \operatorname{coth}(\tau u)} .
\end{aligned}
$$

So, the heat kernel $\kappa_{u, \tau}, u>0$, of $L_{\tau}$ is given by

$$
\kappa_{u, \tau}(z, w)=\frac{1}{4 \pi} \frac{\tau}{\sinh (\tau u)} e^{-\frac{1}{4}|\tau||z-w|^{2} \operatorname{coth}(\tau u)} e^{i \frac{\tau}{4}[z, w]}, \quad z, w \in \mathbb{C} .
$$


5. The Green function and global hypoellipticity of $L_{\tau}$. We can obtain a formula for the Green function $G_{\tau}$ of the twisted Laplacian $L_{\tau}$ by integrating the heat kernel of $L_{\tau}$ from 0 to $\infty$ with respect to time $u$. Indeed, for all $z$ and $w$ in $\mathbb{C}$, we get

$$
\begin{aligned}
G_{\tau}(z, w) & =\frac{1}{4 \pi}\left(\int_{0}^{\infty} \frac{\tau}{\sinh (\tau u)} e^{-\frac{1}{4}|\tau||z-w|^{2} \operatorname{coth}(\tau u)} d u\right) e^{i \frac{\tau}{4}[z, w]} \\
& =\frac{1}{4 \pi}\left(\int_{1}^{\infty} \frac{1}{\left(v^{2}-1\right)^{1 / 2}} e^{-\frac{1}{4}|\tau||z-w|^{2} v} d v\right) e^{i \frac{\tau}{4}[z, w]} \\
& =\frac{1}{4 \pi} K_{0}\left(\frac{1}{4}|\tau||z-w|^{2}\right) e^{i \frac{\tau}{4}[z, w]}
\end{aligned}
$$

where $K_{0}$ is the modified Bessel function of order 0 given by

$$
K_{0}(x)=\int_{0}^{\infty} e^{-x \cosh \delta} d \delta, \quad x>0 .
$$

Using the Green function $G_{\tau}$ and the proof of Theorem 6.1 in [15], we have the following result on the global hypoellipticity of $L_{\tau}, \tau \in \mathbb{R} \backslash\{0\}$.

THEOREM 5.1. For every $\tau$ in $\mathbb{R} \backslash\{0\}$, the twisted Laplacian $L_{\tau}$ is globally hypoelliptic in the sense that

$$
u \in \mathcal{S}^{\prime}\left(\mathbb{R}^{2}\right), L_{\tau} u \in \mathcal{S}\left(\mathbb{R}^{2}\right) \Rightarrow u \in \mathcal{S}\left(\mathbb{R}^{2}\right),
$$

where $\mathcal{S}^{\prime}\left(\mathbb{R}^{2}\right)$ is the space of all tempered distributions on $\mathbb{R}^{2}$.

REMARK 5.2 In fact, it is proved in [5] that for every $\tau$ in $\mathbb{R} \backslash\{0\}$, the twisted Laplacian $L_{\tau}$ is also globally hypoelliptic in Gelfand-Shilov spaces. More precisely, let $\mu$ and $\nu$ be positive real numbers such that $\mu+\nu \geq 1$. Then

$$
u \in \mathcal{S}^{\prime}\left(\mathbb{R}^{2}\right), L_{\tau} u \in \mathcal{S}_{\nu}^{\mu}\left(\mathbb{R}^{2}\right) \Rightarrow u \in \mathcal{S}_{\nu}^{\mu}\left(\mathbb{R}^{2}\right) .
$$

The paper [6] contains similar results for degenerate elliptic operators that include the twisted Laplacians.

6. Liouville's theorems for the sub-Laplacian. The following theorem is a version of Liouville's theorem for the sub-Laplacian $\mathcal{L}$ on the Heisenberg group $\mathbb{H}$.

TheOREM 6.1. Let $u \in \mathcal{S}^{\prime}(\mathbb{H})$ be such that

$$
(\mathcal{L} u)(z, t)=0, \quad(z, t) \in \mathbb{H},
$$

and $\check{u}(z, \tau)$ is a tempered function of $\tau$ on $\mathbb{R}$ for each fixed $z$ in $\mathbb{C}$, where $\check{u}$ is the inverse Fourier transform of $u$ with respect to the "second" variable $t$. Then

$$
u(z, t)=0, \quad(z, t) \in \mathbb{H} .
$$

Proof. Using a result of Hörmander in [7], $u$ is a tempered distribution on $\mathbb{H}$ that is given by a $C^{\infty}$ function on $\mathbb{H}=\mathbb{C} \times \mathbb{R}$. So, by (2), we see that for almost all $\tau$ in $\mathbb{R}$,

$$
\left(L_{\tau} u^{\tau}\right)(z)=(\mathcal{L} u)^{\tau}(z)=0, \quad z \in \mathbb{C} .
$$

By Theorem 5.1 on the global hypoellipticity of $L^{\tau}, \tau \in \mathbb{R} \backslash\{0\}$,

$$
\check{u}(z, \tau)=u^{\tau}(z)=0, \quad z \in \mathbb{C},
$$


for almost all $\tau \in \mathbb{R}$. Hence

$$
u(z, t)=0, \quad(z, t) \in \mathbb{H},
$$

as asserted.

REMARK 6.1. The assumption that $\check{u}(z, \tau)$ is a tempered function of $\tau$ on $\mathbb{R}$ for each fixed $z$ in $\mathbb{C}$ cannot be dropped completely. As an example, let $u$ be the function on $\mathbb{H}$ given by

$$
u(z, t)=1, \quad(z, t) \in \mathbb{H} .
$$

Then $(\mathcal{L} u)(z, t)=0,(z, t) \in \mathbb{H}$, but $u$ is a tempered distribution on $\mathbb{H}$ such that $\check{u}(z, \tau)$ is the Dirac delta in $\tau$ for all $z$ in $\mathbb{C}$.

We can give another version of Liouville's theorem to cover the example in Remark 6.1 .

THEOREM 6.2. Every bounded harmonic function $u$ of the form

$$
u(z, t)=v(z) e^{-i m t}, \quad(z, t) \in \mathbb{H},
$$

for the sub-Laplacian $\mathcal{L}$ on the Heisenberg group $\mathbb{H}$, where $m$ is a real number, has to be a constant function.

Proof. Using the formula (1) for the sub-Laplacian $\mathcal{L}$, we get

$$
(\mathcal{L} u)(z, t)=-(\Delta v)(z) e^{-i m t}+\frac{1}{4}|z|^{2} v(z) m^{2} e^{-i m t}-i(N v)(z) m e^{-i m t}=0
$$

for all $(z, t)$ in $\mathbb{H}$. So, using the formula (2) for the twisted Laplacians $L_{\tau}, \tau \in \mathbb{R} \backslash\{0\}$, we get

$$
L_{m} v=0
$$

if $m \neq 0$ and

$$
L_{0} v=-\Delta v=0 .
$$

By the global hypoellipticity of the twisted Laplacians given by Theorem 5.1, we get $v=0$ if $m \neq 0$. If $m=0$, then by the classic Liouville's theorem for the Laplacian $\Delta$, we see that $v$ has to be a constant function and this completes the proof of the theorem.

Acknowledgments. This research has been supported by the Natural Sciences and Engineering Research Council of Canada.

\section{References}

[1] C. Berenstein, D.-C. Chang and J. Tie, Laguerre Calculus and Its Applications on the Heisenberg Group, American Mathematical Society and International Press, 2001.

[2] P. Boggiatto, E. Buzano and L. Rodino, Global Hypoellipticity and Spectral Theory, Akademie Verlag, 1996.

[3] O. Calin, D.-C. Chang and P. Greiner, Geometric Analysis on the Heisenberg Group and Its Generalizations, American Mathematical Society and International Press, 2007.

[4] W. L. Chow, Über Systeme von linearen partiellen Differentialgleichungen erster Ordnung, Math. Ann. 117 (1939), 98-105. 
[5] A. Dasgupta and M. W. Wong, Essential self-adjointness and global hypoellipticity of the twisted Laplacian, Rend. Sem. Mat. Univ. Politec. Torino 66 (2008), 43-53.

[6] T. Gramchev, S. Pilipović and L. Rodino, Classes of degenerate elliptic operators in Gelfand-Shilov spaces, in: New Developments in Pseudo-Differential Operators, L. Rodino and M. W. Wong (eds.), Birkhäuser, 2009, 15-31.

[7] L. Hörmander, Hypoelliptic second order differential equations, Acta Math. 119 (1967), 147-171.

[8] P. R. Popivanov, A link between small divisors and smoothness of the solutions of a class of partial differential equations, Ann. Global Anal. Geom. 1 (1983), 77-92.

[9] M. A. Shubin, Pseudodifferential Operators and Spectral Theory, Springer-Verlag, 1987.

[10] S. Thangavelu, Harmonic Analysis on the Heisenberg Group, Birkhäuser, 1998.

[11] S. Thangavelu, An Introduction to the Uncertainty Principle: Hardy's Theorem on Lie Groups, Birkhäuser, 2004.

[12] M. W. Wong, Weyl Transforms, Springer-Verlag, 1998.

[13] M. W. Wong, The heat equation for the Hermite operator on the Heisenberg group, Hokkaido Math. J. 34 (2005), 393-404.

[14] M. W. Wong, Weyl transforms and a degenerate elliptic partial differential equation, Proc. R. Soc. London A 461 (2005), 3863-3870.

[15] M. W. Wong, Weyl transforms, the heat kernel and Green function of a degenerate elliptic operator, Ann. Global Anal. Geom. 28 (2005), 271-283. 
